\title{
Non-Aqueous Continuous-Flow Electrophoresis (NACFE): Separation Complement for Continuous-Flow Organic Synthesis
}

\author{
Nikita A. Ivanov, Yimo Liu, Sven Kochmann and Sergey N. Krylov*
}

\begin{abstract}
Continuous-flow organic synthesis naturally requires continuous-flow separation of reaction components. The most common continuous-flow separation approach is liquid-liquid extraction based on differential distribution of molecules between organic and aqueous phases. This approach has limited selectivity; it can hardly separate different hydrophobic organic molecules from each other. Continuous-flow electrophoresis can facilitate much more selective separation in a single phase, but it is currently limited to aqueous electrolytes which are incompatible with many hydrophobic organic molecules. Further, water electrolysis in aqueous electrolytes results in generation of large volumes of gas making steady-state operation a major technical challenge. Here, we introduce non-aqueous continuous-flow electrophoresis (NACFE) in which the electrolyte is a solution of an organic salt in an aprotic organic solvent. We demonstrate that NACFE can maintain stable separation of multiple species during 10 hours. The non-aqueous nature of NACFE and its ability to support steady-state operation make it suitable for its incorporation into continuous-flow organic synthesis.
\end{abstract}

Continuous-flow organic synthesis has a number of important advantages over its batch counterpart. ${ }^{[1]}$ Continuous-flow separation of multiple reaction components (e.g. an intermediate, an excess reactant, and a catalyst) from each other is often required between the stages of continuous-flow organic synthesis. ${ }^{\left[{ }^{2]}\right.}$ Liquidliquid extraction that segregates molecules through their partitioning between organic and aqueous phases is the most common continuous-flow separation method. ${ }^{[3]}$ Yet, it can hardly separate hydrophobic organic molecules with similar partition coefficients from each other. ${ }^{[4]}$ Continuous-flow electrophoresis (CFE), which also called free-flow electrophoresis, can support much more selective separation. ${ }^{[5]}$ Its ability to separate multiple molecular streams in a single phase could potentially facilitate its seamless integration with continuous-flow organic synthesis. However, practical CFE has been so far limited to aqueous electrolytes; with an exception of a single work reporting the use of a non-aqueous electrolyte including a protic organic solvent (methanol). ${ }^{[6]}$ Aqueous electrolytes are incompatible with continuous-flow organic synthesis, as the synthesis often involves reaction components insoluble in water. ${ }^{[7]}$ An additional problem with aqueous electrolytes is intensive gas formation during water electrolysis; hardly avoidable accumulation of gas bubbles in the separation chamber makes steady-state operation of CFE a technical challenge (protic organic solvents suffer from the same problem). ${ }^{[8]}$ On the contrary, non-aqueous continuous-flow electrophoresis (NACFE) could potentially use aprotic organic

[*] Centre for Research on Biomolecular Interactions and Department of Chemistry, York University, Toronto, Ontario M3J 1P3, Canada E-mail: skrylov@yorku.ca

Homepage: http://www.yorku.ca/skrylov/

ORCIDs:

Nikita A. Ivanov: 0000-0002-0842-6626

Sven Kochmann: 0000-0001-7423-4609

Sergey N. Krylov: 0000-0003-3270-2130

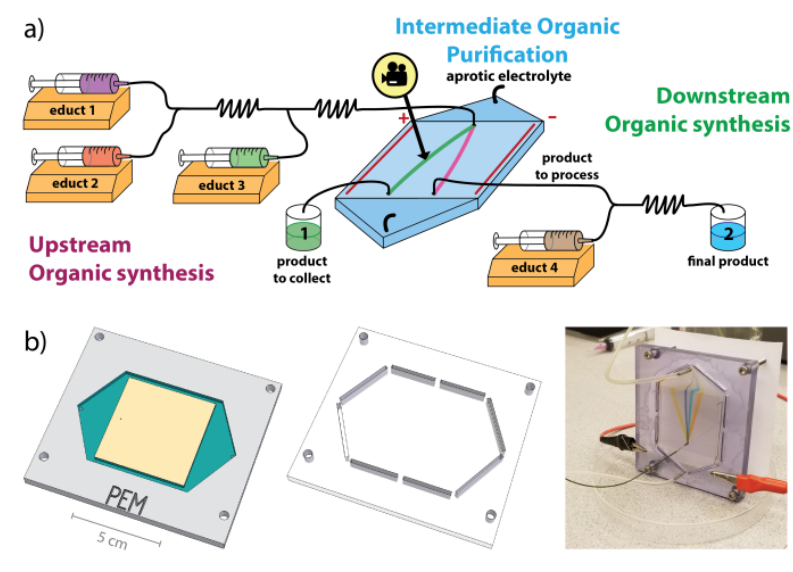

Figure 1. a) Schematic of NACFE seamlessly integrated between two stages (upstream and downstream) of continuous-flow organic synthesis. b) Geometry of the bottom part (left) and top part (middle) of the NACFE chip used in this study as well as its photo in operation (right).

solvents which are not only compatible with solvents utilized in continuous-flow organic synthesis but also could minimize gas formation and make separation stable without elaborate technical solutions. Owing to these two anticipated advantages, NACFE utilizing aprotic organic solvents appears to be a highly attractive separation complement for continuous-flow organic synthesis (Figure 1a). Devices for NACFE are simple and can be easily custom fabricated (Figure 1b). Therefore, it is rather surprizing that there have been no reports on this technique while aprotic nonaqueous electrolytes, e.g. based on cyclic carbonates, are widely used in batteries, ${ }^{[9]}$ and have been used in discontinuous separation by capillary electrophoresis. ${ }^{[10]}$ The goal of this work was to prove the feasibility of NACFE with aprotic electrolytes and test its suitability for steady-state separation of multiple molecular streams in organic phase.

Continuous-flow separation (as an integral part of continuousflow synthesis) must operate under steady-state conditions. We, thus, aimed at developing steady-state NACFE confirmed by stable uninterrupted operation during e.g. a 10-h shift. Separation instability in CFE is caused by gradually growing distortion of the hydrodynamic flow and/or electric field during the course of operation. The major and most persistent cause of such distortion is hardly-avoidable accumulation of gas bubbles in the device. ${ }^{[8]}$ The only proven long-term solution for this problem is bubble evacuation to the atmosphere through an open-electrolyte approach. ${ }^{[8 a]}$ This approach should, however, be avoided for nonaqueous electrolytes due to safety concerns. Thus, we limited ourselves to closed NACFE devices. In NACFE, one can foresee another potential source of growing distortion of hydrodynamic flow: deterioration of the electrophoretic device under the influence of an organic solvent. Hence, a NACFE device must be made of a solvent-resistant material. We chose propylene carbonate (PC) as an aprotic organic solvent, and had to use a device material resistant to it. While silica glass is arguably the best choice of a solvent-resistant material, making prototype 


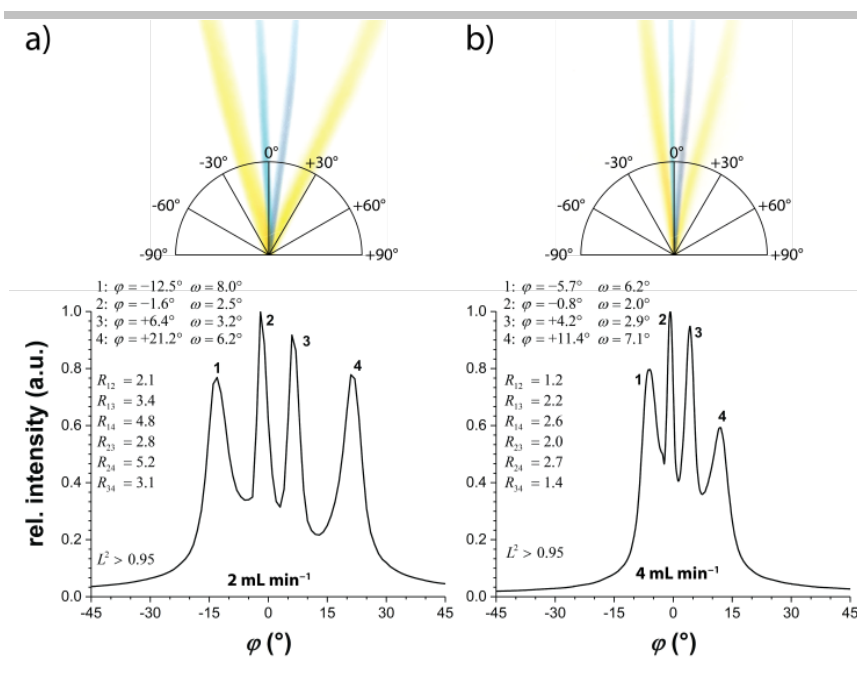

Figure 2. Angulagrams of NACFE of fluorescein (1), a-naphtholbenzein (2), Sudan black B (3), and DMAS (4) (1.25 mM each) in $30 \mathrm{mM}$ TBAA in PC at two different electrolyte flowrates: a) 2 and b) $4 \mathrm{~mL} / \mathrm{min}$. The values in the graph are stream deflection $(\varphi)$, stream width $(\omega)$, stream linearity $\left(L^{2}\right)$, and resolution of stream $n$ from stream $m\left(R_{\mathrm{nm}}\right)$. NACFE was run with $E=27.3 \mathrm{~V} / \mathrm{cm}$ $(I=8.3 \mathrm{~mA})$ and a sample flowrate of $2 \mu \mathrm{L} / \mathrm{min}$. The anode and cathode are towards negative and positive angles, respectively.

NACFE devices of HF-etched glass is not as practical as making them of mechanically-machined plastics. We tested a set of 18 plastics, commercially available in sheets, for their machinability, optical clarity, and resistance to PC (Table S1). Three of them, fluorinated ethylene propylene, polyvinyl chloride (PVC) Type I, and polysulfone, were found potentially suitable based on these three parameters. Of these three plastics, we chose PVC Type I for its optical clarity, cost efficiency, and full suitability for devicefabrication procedures previously developed for poly(methyl methacrylate). ${ }^{[11]}$ A NACFE chip of a basic geometry (Figures $1 \mathrm{~b}$ and S1) was fabricated and used for all experiments described below.

A key component of any electrolyte is a charge carrier, which is typically a well-soluble non-reactive salt giving a free cation and a free anion upon dissociation. Two types of organic salts have been previously used as charge carriers in non-aqueous aprotic electrolytes in capillary electrophoresis: tetraalkylammonium salts and imidazolium salts (ionic liquids). ${ }^{[12]}$ Their use in capillary electrophoresis did not guarantee transferability to NACFE. In contrast to capillary electrophoresis, electrodes in NACFE are inside the separation chamber making NACFE susceptible to instability associated with electrochemistry of electrolyte components. In addition, capillary electrophoresis runs take only a few minutes, and long-term stability is not a requirement in contrast to NACFE. Therefore, we first tested NACFE for longterm stability of electric current and optical properties of the chip. The experiment was done for two electrolytes: solutions of tetrabutylammonium acetate (TBAA) and imidazolium ethyl sulfate in PC. We found that the electrical current was stable for TBAA during a 10-h run (Figure S2). No gas bubble accumulation was evident. Minor precipitation could be noticed at the cathode side of the NACFE chip likely due to an electrochemical reaction involving tetrabutylammonium. This precipitate did not affect optical clarity of the chip. In contrast, we found the excessive formation of a brown precipitate at the cathode side of the chip for the imidazolium electrolyte (Figure S3). The precipitate was most likely an insoluble product of an electrochemical reaction involving imidazolium. ${ }^{[13]}$ This precipitate affected optical clarity of the chip and could interfere with optical detection; accordingly, we ruled out imidazolium-based electrolytes from our further consideration. Hence, a solution of TBAA in PC was chosen as a default electrolyte for the rest of this NACFE study.

Next, we tested separation of multiple molecular streams in NACFE (Figure 2). As molecules to be separated we used 2-[4(dimethylamino)styryl]-1-methylpyridinium (DMAS), Sudan black B, $\alpha$-naphtholbenzein, and fluorescein (the first three are hydrophobic and poorly soluble in water). All of them are chromophores visible to the naked eye, which facilitated easy detection of their streams in this proof-of-feasibility work.

The quality of NACFE was assessed using a recently introduced approach based on angulagram representation of molecular stream separation and four quantitative characteristics: stream deflection, stream width, stream linearity, and resolution of two streams. ${ }^{[14]}$ Images of molecular stream in NACFE were recorded with a consumer photo camera. These images were processed automatically to construct angulagrams and compute the quantitative characteristics of the streams (see Supporting Information for details on these procedures and corresponding custom-designed software).

All molecular streams shown in Figure 2 were deflected as predicted by the previously proposed separation mechanism based on heteroconjugation. ${ }^{[15]}$ Briefly, small anions (e.g. acetate) form heteroconjugates with hydrogen-bond donors. The effective charge of a heteroconjugate is negative and its magnitude depends on the degree of heteroconjugation; this dependency is advantageous and allows, for instance, the separation of different phenols. In our case, $\alpha$-naphtholbenzein (phenol) and fluorescein (carboxylic acid and phenol) formed negatively charged heteroconjugates, which were deflected towards the anode. DMAS is not a hydrogen-bond donor but possesses one quaternary nitrogen atom with a positive charge. Hence, DMAS was deflected towards the cathode. Sudan black B has no positive charge and is assumed to be a very weak hydrogenbond donor, which, however, still can experience a low degree of heteroconjugation. Therefore, its stream was expected to be deflected slightly towards the anode, i.e. negative angles in the angulagram in Figure 2. In fact, it was deflected towards the cathode, i.e. positive angles. This small deflection was likely due to the presence of the electroosmotic flow ${ }^{[16]}$ (from anode to cathode) and affected deflection of other streams as well. Ideally, one would compare the experimental deflection angles to theoretical ones; ${ }^{[14]}$ however, the theory for calculating electrophoretic mobilities (which define deflection angles) is not straightforwardly applicable to non-aqueous electrophoresis.

All streams in Figure 2 are linear $\left(L^{2}>0.95\right)$ and narrow $\left(<10^{\circ}\right.$ in width). The worst stream resolution $(R>1.2)$ in Figure 2 is still sufficient for collecting any individual stream without overlap with any other stream $(R \geq 1.0$ is our threshold for collectable streams $\left.{ }^{[14]}\right)$.

Increasing the concentration of the charge carrier in the electrolyte expectedly led to improved separation confirmed by decreasing stream width and increasing linearity (Figure 3). Unsurprisingly, no separation could be observed when the electrolyte was replaced with the pure organic solvent without any charge carrier. Adding a charge carrier at a concentration (3.3 mM) similar to the analyte concentration $(1.67 \mathrm{mM})$ resulted in one broad stream (width: $9.8^{\circ}$ ) in which the individual analytes started to separate. Increasing the charge carrier concentration from 3.3 to $10 \mathrm{mM}$ (an order of magnitude higher than the analyte concentration) turned this broad stream into individual narrow ones (widths of 4.4 to $6.8^{\circ}$ ); further increase to $30 \mathrm{mM}$ narrowed the 


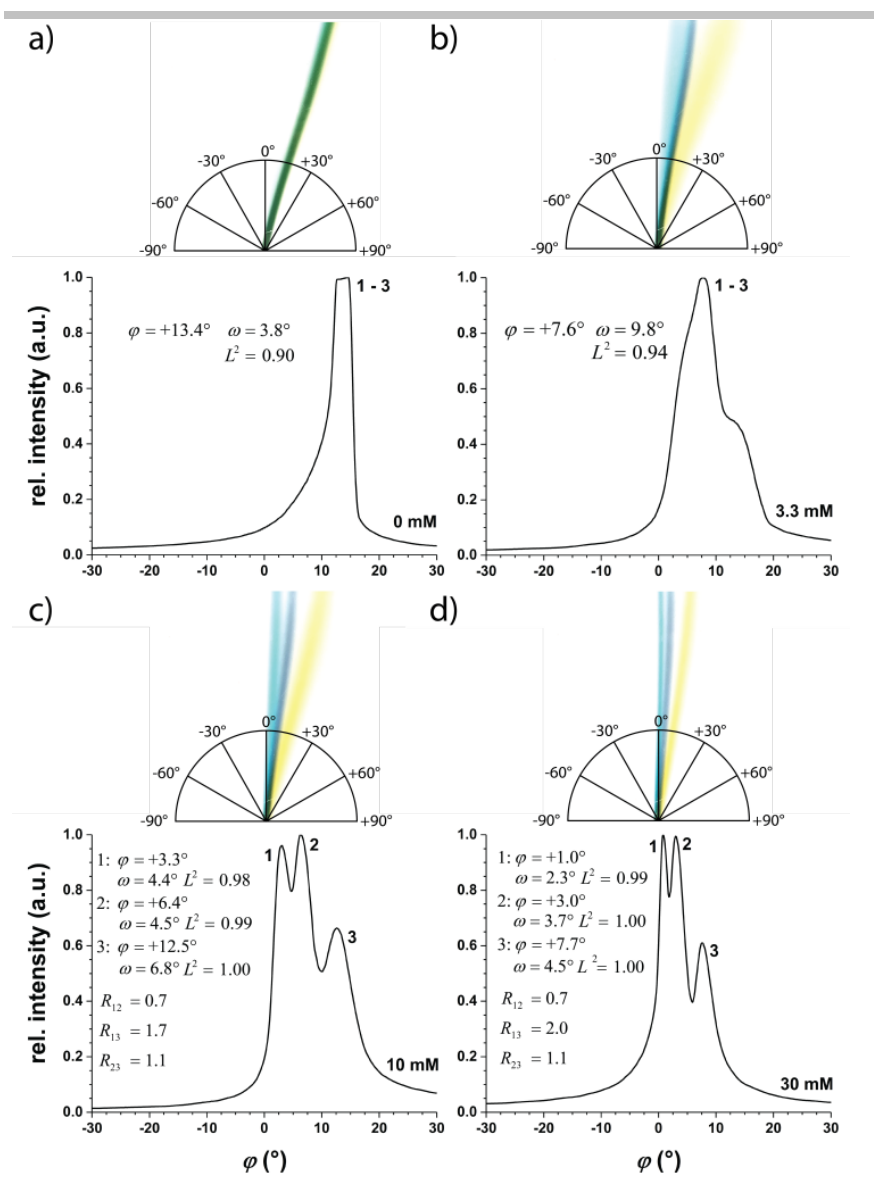

Figure 3. Angulagrams of NACFE of $\alpha$-naphtholbenzein (1), Sudan black B (2), and DMAS (3) (1.67 mM each) under various concentrations of TBAA in PC: a) 0, b) 3.3 , c) 10 and d) $30 \mathrm{mM}$. Given are stream deflection $(\varphi)$, stream width $(\omega)$, stream linearity $\left(L^{2}\right)$, and resolution of stream $n$ from stream $m\left(R_{\mathrm{nm}}\right)$. $E=27.3 \mathrm{~V} / \mathrm{cm}$ was used in all experiments and resulted in currents of 0.07 (a), 1.26 (b), 3.69 (c), and $9.9 \mathrm{~mA}$ (d). The flowrates were $3 \mathrm{~mL} / \mathrm{min}$ for the electrolyte and $2 \mu \mathrm{L} / \mathrm{min}$ for the sample. The anode and cathode are towards negative and positive angles, respectively.

streams even more (widths of 2.3 to $4.5^{\circ}$ ). The carrier concentration had only minimal effect on the linearity; linearity increased from 0.90 to 1.00 with carrier concentration increasing from 0 to $30 \mathrm{mM}$. Increase in the carrier concentration progressively suppresses the electroosmotic flow directed towards the cathode. ${ }^{[17]}$ Hence, stream deflections changed towards the anode with increasing carrier concentration. For instance, the stream deflection of Sudan black B changed from +13.4 to $+3.0^{\circ}$ (i.e. became less deflected) when increasing the carrier concentration from 0 to $30 \mathrm{mM}$. The quality of separation depended on the nature of the anion in the charge carrier. A weakbase anion (e.g. hydrogen sulfate) could not support the separation of $\alpha$-naphtholbenzein from Sudan black B, while a strong base (e.g. acetate) could separate them (Figure S4). All results discussed above are consistent with the aforementioned separation mechanism in NACFE, in which heteroconjugation of electrolyte anions with hydrogen-bond donors plays a key role (see above). ${ }^{[15]}$

Finally, we examined whether steady-state NACFE could be maintained (e.g. stable separation during a 10-h shift), which is the key requirement for integrating NACFE with continuous-flow synthesis. Three hydrophobic analytes were used in this experiment: $\alpha$-naphtholbenzein, Sudan black B, and DMAS. The electrolyte was recycled roughly every $2.5 \mathrm{~h}$ to minimize material waste; we did not interrupt separation for electrolyte recycling. Our
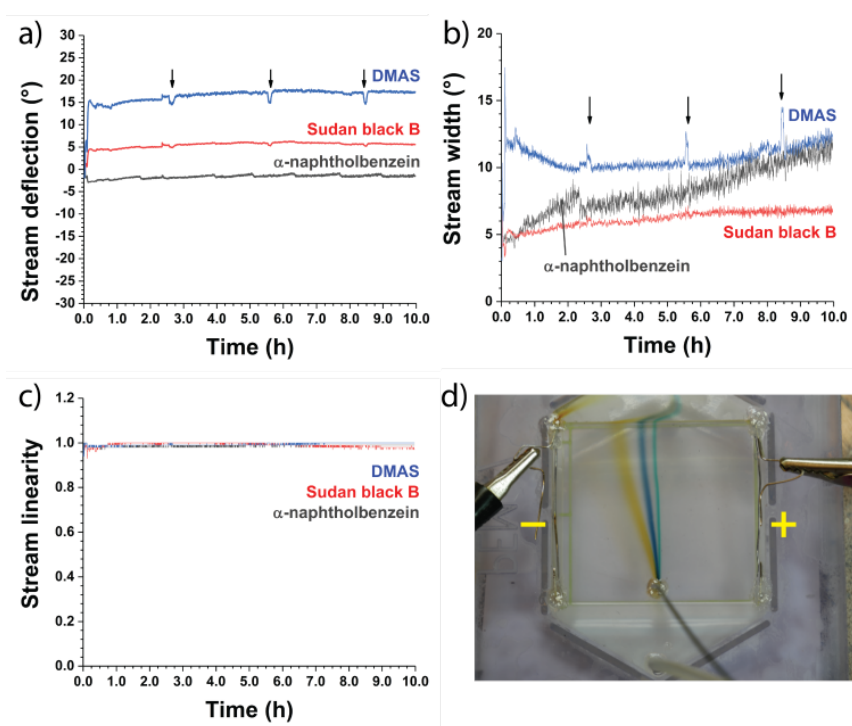

Figure 4. a-c) Stream parameters and d) an averaged photo (3587 images were integrated for visual assessment of separation stability) of 10-h NACFE of a-naphtholbenzein, Sudan black B, and DMAS (1.67 mM each) in $30 \mathrm{mM}$ TBAA in PC. Recycling was done roughly every $2.5 \mathrm{~h}$ (marked with arrows).

results show no significant deterioration in stream deflection, width or linearity during the 10-h NACFE with the exception of stream width for $\alpha$-naphtholbenzein which drifted from 5 to $12^{\circ}$ (Figure 4). This degree of stream widening, however, would not affect significantly stream collection for the magnitudes of resolution and stability of deflection demonstrated.

In the frame of the present work, we also explored the option of using acetonitrile instead of PC in NACFE. Our results demonstrate that separation in acetonitrile-based electrolytes is possible in principle (Figure S5). However, electrolytes based on PC allowed lower flowrates (due to lesser gas bubble formation) and higher electric fields (due to lower currents and Joule heating). Furthermore, PC is a less toxic, less volatile, and more viscous solvent than acetonitrile. ${ }^{[18]}$ Thus, we did not further investigate acetonitrile in the frame of this work.

In conclusion, we proved the feasibility of NACFE with an aprotic electrolyte, namely TBAA in PC, by demonstrating steadystate separations of multiple molecular streams. This proven feasibility should stimulate efforts to implement integrated NACFE/continuous-flow synthesis. Here, we would like to outline what is required for such an implementation. First, any NACFE device must have outputs for multiple molecular streams; such devices have been successfully fabricated and used in the past. ${ }^{[19]}$ Second, the optimization of device operation, e.g. adjustment of electric field and flowrate, requires visualization/ detection of molecular streams. Most organic molecules are not chromophores visible to the naked eye but absorb UV light; therefore, UVimaging of a large area of the NACFE chip is required. Belder and co-authors have recently demonstrated deep-UV fluorescence imaging of UV-absorbing molecular streams in a small CFE chip. ${ }^{[20]}$ This is a highly promising approach towards UV-imaging of larger chips. However, significant increases in scanning speed and covered area are required to apply this approach to real-time imaging of larger chips. We foresee that solving this challenging detection issue will open the route for practical use of NACFE in combination with continuous-flow synthesis. 


\section{Conflict of interest}

The authors declare no conflict of interest.

\section{Acknowledgements}

This work was supported by NSERC grant (STPG-P 521331-2018) to SNK. The authors thank Dr. Boris I. Gorin for valuable discussions.

Keywords: continuous-flow organic synthesis • continuous-flow separation • non-aqueous continuous-flow electrophoresis $\bullet$ cyclic carbonates $\bullet$ tetraalkylammonium salts

[1] a) J. S. Moore, K. F. Jensen, Org. Process Res. Dev. 2012, 16, 1409-1415; b) Á. Tajti, N. Tóth, E. Bálint, G. Keglevich, J. Flow. Chem. 2018, 8, 11-19; c) K. F. Jensen, AIChE J. 2017, 63, 858-869; d) C. Len, S. Bruniaux, F. Delbecq, S. V. Parmar, Catalysts 2017, 7, 146; e) J. A. M. Lummiss, P. D. Morse, R. L. Beingessner, T. F. Jamison, Chem. Rec. 2017, 17, 667-680; e) S. G. Koenig, B. Dillon, Curr. Opin. Green Sustainable Chem. 2017, 7, 56-59; f) G. S. Fleming, A. B. Beeler, J. Flow Chem. 2017, 7, 124-128.

[2] a) P. L. Heider et al., Org. Process Res. Dev. 2014, 18, 402-409; b) P. Bana, R. Örkényi, K. Lövei, Á. Lakó, G. I. Túrós, J. Éles, F. Faigl, I. Greiner, Biorg. Med. Chem. 2017, 25, 6180-6189; c) J. Britton, C. L. Raston, Chem. Soc. Rev. 2017, 46, 1250-1271

[3] a) J. Zhang, K. Wang, A. R. Teixeira, K. F. Jensen, G. Luo, Annu. Rev. Chem. Biomol. Eng. 2017, 8, 285-305; b) E. Y. Kenig, Y. Su, A. Lautenschleger, P. Chasanis, M. Grünewald, Sep. Purif. Technol. 2013, 120, 245-264.

[4] J. G. Kralj, H. R. Sahoo, K. F. Jensen, Lab Chip 2007, 7, 256-263.

[5] B. R. Fonslow, M. T. Bowser, Anal. Chem. 2006, 78, 8236-8244.

[6] N. W. Frost, M. T. Bowser, Lab Chip 2010, 10, 1231-1236.
[7] P. L. Suryawanshi, S. P. Gumfekar, B. A. Bhanvase, S. H. Sonawane, M. S. Pimplapure, Chem. Eng. Sci. 2018, 189, 431-448.

[8] a) F. J. Agostino, L. T. Cherney, V. Galievsky, S. N. Krylov, Angew. Chem 2013, 125, 7397-7401; b) D. Kohlheyer, J. C. T. Eijkel, S. Schlautmann, A. van den Berg, R. B. M. Schasfoort, Anal. Chem. 2008, 80, 4111-4118; c) A. C. Johnson, M. T. Bowser, Lab Chip 2018, 18, 27-40; d) C. Herzog, G. F. W. Jochem, P. Glaeser, S. Nagl, Microchim. Acta 2015, 182, 887-892; e) P. Novo, M. Dell'Aica, M. Jender, S. Höving, R. P. Zahedi, D. Janasek, Analyst 2017, 142, 4228-4239; f) F. J. Agostino, S. N. Krylov, Trends Anal. Chem 2015, 72, 68-79.

[9] S-I. Tobishima, A. Yamaji, Electrochim. Acta 1984, 29, 267-271.

[10] J. Muzikar, T. van de Goor, B. Gaš, E. Kenndler, Anal. Chem. 2002, 74, $428-433$

[11] a) F. J. Agostino, C. J. Evenhuis, S. N. Krylov, J. Sep. Sci. 2011, 34, 556-564; b) S. Kochmann, S. N. Krylov, Lab Chip 2017, 17, 256-266.

[12] (a) R. Kuldvee, M. Vaher, M. Koel, M. Kaljurand, Electrophoresis 2003, 25 1627-1634; (b) E. Kenndler, J. Chromatogr. A 2014, 1335, 16-30.

[13] D. T. Walker, C. D. Douglas, B. J. MacLean, Can. J. Chem. 2009, 87, 729-737.

[14] S. Kochmann, S. N. Krylov, Anal. Chem. 2018, 90, 9504-9509.

[15] a) T. Okada, J. Chromatogr. A 1997, 771, 275-284; b) S. P. Porras, R. Kuldvee, S. Palonen, M-L. Riekkola, J. Chromatogr. A 2003, 990, 35-44.

[16] a) V. Tandon, S. K. Bhagavatula, W. C. Nelson, B. J. Kirby, Electrophoresis 2008, 29, 1092-1101; b) D. L. Pugmire, E. A. Waddell, R. Haasch, M. J. Tarlov, L. E. Locascio, Anal. Chem. 2002, 74, 871-878; c) W. Schützner, E. Kenndler, Anal. Chem. 1992, 64, 1991-1995.

[17] M. F. M. Tavares, V. L. McGuffin, Anal. Chem. 1995, 67, 3687-3696.

[18] a) Propylene carbonate, MSDS No. O43314, Thermo Fisher Scientific, https:// www.fishersci.com/store/msds?partNumber=043314\&vendorId=VN00033897 \&countryCode=CA (accessed Mar 08, 2019). b) Acetontrile, MSDS No. A9981, Thermo Fisher Scientific, https://www.fishersci.ca/store/msds?partNumber= A9981\&countryCode $=$ CA\&language $=$ en (accessed Mar 08, 2019).

[19] a) B. R. Fonslow, M. T. Bowser, Anal. Chem. 2005, 77, 5706-5710; b) M. Nissum, A. L. Foucher, Expert Rev. Proteomics 2008, 5, 571-587; c) S. Köhler, C. Benz, H. Becker, E. Beckert, V. Beushausen, D. Belder, RSC Adv. 2012, 2, 520-525.

[20] a) S. Köhler, S. Nagl, S. Fritzsche, D. Belder, Lab Chip 2012, 12, 458-463; b) S. A. Pfeiffer, B. M. Rudisch, P. Glaeser, M. Spanka, F. Nitschke, A. A Robitzki, C. Schneider, S. Nagl, D. Belder, Anal. Bioanal. Chem. 2018, 410, 853-862. 